\title{
General theorem on the Schrödinger equation
}

\author{
Xiang Rong Wang \\ Department of Physics, Hong Kong University of Science and Technology, Clear Water Bay, Kowloon, Hong Kong \\ and School of Physics and Astronomy, University of Minnesota, Minneapolis, Minnesota 55455
}

(Received 1 May 1992)

\begin{abstract}
Two discrete energy spectra of a particle moving in two different potential wells are compared. We find a general property: If one potential well $V_{1}(\mathbf{r})$ is less than or equal to the other potential well $V_{2}(\mathbf{r})$ everywhere in space, i.e., $V_{1}(\mathbf{r}) \leq V_{2}(\mathbf{r})$ for all $\mathbf{r}$, then the $n$th eigenvalue of the particle in the first well, $V_{1}$, is always not greater than the $n$th eigenvalue of the particle in the second well, $V_{2}$.
\end{abstract}

PACS number(s): 03.65.Ge, 02.10. $+\mathrm{w}, 02.90 .+\mathrm{p}$

It is unnecessary, nowadays, to point out the importance of the Schrödinger equation to disciplines of science and technology. In principle, one can understand any natural phenomenon if one can solve the corresponding Schrödinger equations. Unfortunately, the Schrödinger equation can only be analytically solved in a few special cases. Therefore, it is very important to know the properties of the equation.

There are many theorems and properties [1,2] which help us to understand various aspects of the Schrödinger equation. One of them concerns the ground state of a particle in two different potential wells: It is well known that if one well $V_{1}$ is less than or equal to the second well $V_{2}$ everywhere in space, the ground-state energy in the well $V_{1}$ will not always be higher than that in the well $V_{2}$. This property can be easily shown using the variational theorem, i.e., energy expectation of a trial state is always greater than or equal to the ground state of the system. However, to the best of our knowledge, there is nothing in the literature comparing levels other than the ground state. It is the purpose of this letter to show that a similar property is also true for the excited states.

Let us consider the energy spectrum of a particle moving in a potential well, $V(\mathbf{r})$. According to quantum mechanics, the spectrum well be governed by the timeindependent Schrödinger equation

$$
\mathscr{H} \phi(\mathbf{r})=\varepsilon \phi(\mathbf{r}),
$$

with proper boundary conditions; here, $\mathscr{H}=\widehat{T}+V(\mathbf{r})$ is called the Hamiltonian, and $\hat{T}$ is the kinetic-energy operator which is defined as $\hat{T}=-(\hbar / 2 m) \nabla^{2}$, where $m$ is the mass of the particle. It is well known that $\mathscr{H}$ is Hermitian, its eigenvalues are all real, and its eigenstates can be chosen to be orthogonal. In this letter, we will study the energy spectra of a particle moving in two different potential wells, $V_{1}(\mathbf{r})$ and $V_{2}(\mathbf{r})$. We will show that there exists the following theorem.

Theorem. For two Hamiltonians $H_{1}=\widehat{T}+V_{1}$ and $H_{2}=\widehat{T}+V_{2}$ describing a particle in real potentials $V_{1}(\mathbf{r})$ and $V_{2}(\mathbf{r})$, respectively, let us denote by $\varepsilon_{1, n}$ and $\varepsilon_{2, n}$ their $n$th eigenvalues; then $\varepsilon_{1, n} \leq \varepsilon_{2, n}$ if $V_{1}(\mathbf{r}) \leq V_{2}(\mathbf{r})$ for all $\mathbf{r}$.

Let us denote $\phi_{1, n}(\mathbf{r})$ and $\phi_{2, n}(\mathbf{r})$ as the $n$th eigenstate of $\mathscr{H}_{1}$ and $\mathscr{H}_{2}$, respectively, i.e.,

$$
\mathscr{H}_{1} \phi_{1, n}(\mathbf{r})=\varepsilon_{1, n} \phi_{1, n}(\mathbf{r})
$$

and

$$
\mathscr{H}_{2} \phi_{2, n}(\mathbf{r})=\varepsilon_{2, n} \phi_{2, n}(\mathbf{r}) .
$$

For any $n$, if we show that we can always find a trial wave function $\varphi(\mathbf{r})$ such that it is orthogonal to the first $n-1$ eigenstates of $\mathscr{H}_{1}$,

$$
\left\langle\varphi(\mathbf{r}) \mid \phi_{1, j}(\mathbf{r})\right\rangle=0 \text { for } j=0, \ldots, n-1,
$$

and satisfies the inequality

$$
\frac{\left\langle\varphi(\mathbf{r})\left|\mathcal{H}_{1}\right| \varphi(\mathbf{r})\right\rangle}{\langle\varphi(\mathbf{r}) \mid \varphi(\mathbf{r})\rangle} \leq \varepsilon_{2, n},
$$

then, according to the variational principle, the theorem is proved.

If we suppose that the wave function

$$
\varphi(\mathbf{r}) \in \mathbb{U}=\left\{\phi_{2,0}, \phi_{2,1}, \ldots, \phi_{2, n}\right\},
$$

a subspace spanned by $\phi_{2,0}, \phi_{2,1}, \ldots, \phi_{2, n}$, it is obvious that

$$
\varepsilon_{1, n} \leq \frac{\left\langle\varphi(\mathbf{r})\left|\mathcal{H}_{1}\right| \varphi(\mathbf{r})\right\rangle}{\langle\varphi(\mathbf{r}) \mid \varphi(\mathbf{r})\rangle} \leq \frac{\left\langle\varphi(\mathbf{r})\left|\mathcal{H}_{2}\right| \varphi(\mathbf{r})\right\rangle}{\langle\varphi(\mathbf{r}) \mid \varphi(\mathbf{r})\rangle} \leq \varepsilon_{2, n},
$$

if $V_{1}(\mathbf{r}) \leq V_{2}(\mathbf{r})$ for any $\mathbf{r}$. Therefore, in order to prove the theorem, it will be sufficient to show that the following statement is true.

Statement. Suppose $\phi_{1, n}(\mathbf{r})$ and $\phi_{2, n}(\mathbf{r})$ are the $n$th eigenstates of Hamiltonian $\mathscr{H}_{1}$ and $\mathscr{H}_{2}$, respectively, it is always possible to find a set of numbers, $C_{0}, C_{1}, \ldots, C_{n}$, which will not all be zeros, such that

$$
\varphi(\mathbf{r})=\sum_{i=0}^{n} C_{i} \phi_{2, i}(\mathbf{r})
$$

will be orthogonal to $\phi_{1, j}(\mathbf{r})$, for $j=0,1, \ldots, n-1$.

Without losing generality [1], we will assume

$$
\left\langle\phi_{\alpha, j}(\mathbf{r}) \mid \phi_{\alpha, k}(\mathbf{r})\right\rangle=\delta_{j, k}, \quad \alpha=1,2 .
$$

Before proving the statement for arbitrary $n$, let us first look at the case when $n=1$. One only needs to show that 
it is possible to find a wave function which is the linear combination of $\phi_{2,0}(\mathbf{r})$ and $\phi_{2,1}(\mathbf{r})$ such that the wave function will be orthogonal to $\phi_{1,0}(\mathbf{r})$. This is obviously true if

$$
\left\langle\phi_{1,0}(\mathbf{r}) \mid \phi_{2,0}(\mathbf{r})\right\rangle=0
$$

or

$$
\left\langle\phi_{1,0}(\mathbf{r}) \mid \phi_{2,1}(\mathbf{r})\right\rangle=0 .
$$

It is also quite clear that

$$
\varphi(\mathbf{r})=\phi_{2,0}(\mathbf{r})-\left(\left\langle\phi_{1,0} \mid \phi_{2,0}\right\rangle /\left\langle\phi_{1,0} \mid \phi_{2,1}\right\rangle\right) \phi_{2,1}(\mathbf{r}),
$$

i.e., $C_{0}=1$ and

$$
C_{1}=-\left\langle\phi_{1,0} \mid \phi_{2,0}\right\rangle /\left\langle\phi_{1,0} \mid \phi_{2,1}\right\rangle
$$

will satisfy the requirement if

$$
\left\langle\phi_{1,0}(\mathbf{r}) \mid \phi_{2,0}(\mathbf{r})\right\rangle \neq 0
$$

and

$$
\left\langle\phi_{1,0}(\mathbf{r}) \mid \phi_{2,1}(\mathbf{r})\right\rangle \neq 0 .
$$

Therefore, the statement is correct for $n=1$. There is a simple geometry analogy in a finite $d$-dimensional space for the case. It is the statement that the intersection of a $(d-1)$-dimensional object and a two-dimensional object in $d$-dimensional space is always nonzero. To be more accurate, the intersection is no less than $(d-1)+2-d=1$ dimension.

For a general $n$, it is easy to see that the question is the existence of a nonzero solution of the linear equations:

$$
\left(\begin{array}{cccc}
a_{0,0} & a_{0,1} & \cdots & a_{0, n} \\
a_{1,0} & a_{1,1} & \cdots & a_{1, n} \\
\vdots & \vdots & \ddots & \vdots \\
a_{n-1,0} & a_{n-1,1} & \cdots & a_{n-1, n}
\end{array}\right)\left(\begin{array}{c}
C_{0} \\
C_{1} \\
\vdots \\
C_{n}
\end{array}\right)=0,
$$

where

$$
a_{j, i} \equiv\left\langle\phi_{1, j}(\mathbf{r}) \mid \phi_{2, i}(\mathbf{r})\right\rangle
$$

Since the matrix is $n \times(n+1)$ order, according to the linear algebra theory [3], it will have nonzero solutions. Therefore, the statement is correct, and the theorem is proved. The geometry analogy of the general case is that the intersection of $n(d-1)$-dimensional objects and one $(n+1)$-dimensional object in a $d$-dimensional space is not zero.

In the proof above, we did not assume that there are no degenerate energy levels, or that the spectra are discrete or continued. We only required that the energy spectrum can be ordered in such a way that $\varepsilon_{\alpha, n} \leq \varepsilon_{\alpha, m}$ if $n<m$, where $\alpha=1$ or 2 . Therefore, the result and proof will not be changed if a degeneracy occurs at a certain eigenvalue, or if the spectra are continued. There is no need to assume that the quantum index $n$ is finite in the proof. One should expect that the result holds when $n$ goes to infinity. In conclusion, we have compared the spectra of a particle in two potential wells, $V_{1}(\mathbf{r})$ and $V_{2}(\mathbf{r})$, respectively, in the case where $V_{1}(\mathbf{r}) \leq V_{2}(\mathbf{r})$ for all $\mathbf{r}$. We find that the $n$th eigenvalue of the particle in the potential $V_{1}$ is always less than or equal to the $n$th eigenvalue of the particle in the potential $V_{2}$.

The author acknowledges stimulating discussions with Professor J. W. Halley, Professor Y. Shapir, Professor C. Campbell, Professor M. Hamermesh, Professor P. Rejto, Professor P. J. Ellis, and Mr. Fei Lou. This work was supported by the Department of Energy through the Corrosion Research Center at University of Minnesota (Grant No. DOE/DE-FG02-84ER45173), by Minnesota University Supercomputer Institute, and by a Sponsored University Research grant from IBM.
[1] Albert Messiah, Quantum Mechanics (North-Holland, Amsterdam, 1965).

[2] Berry Simon, Ann. Phys. 146, 209 (1983); Int. J. Quantum
Chem. 21, 3 (1982).

[3] L. Mirsky, An Introduction to Linear Algebra (Clarendon, Oxford, 1961). 